\title{
REFINED INEQUALITIES ON THE WEIGHTED LOGARITHMIC MEAN
}

\section{Shigeru Furuichi AND Nicuşor Minculete}

Abstract. Inspired by the recent work by R. Pal et al., we give further refined inequalities for a convex Riemann integrable function, applying the standard Hermite-Hadamard inequality. Our approach is different from their one in [9]. As corollaries, we give the refined inequalities on the weighted logarithmic mean and the weighted identric mean. Some further extensions are also given.

Mathematics subject classification (2010): Primary 26D15; Secondary 26B25, 26E60.

Keywords and phrases: Weighted logarithmic mean, weighted identric mean, convex function, HermiteHadamard inequality and operator inequality.

\section{REFERENCES}

[1] P. Cerone And S. S. Dragomir, Ostrowski type inequalities for functions whose derivatives satisfy certain convexity assumptions, Demonstratio Math., 37 (2) (2004) 299-308.

[2] S. S. DRAgomir And R. P. Agarwal, Two inequalities for differentiable mappings and applications to special means of real numbers and to trapezoidal formula, Appl. Math. Lett., 11, (5) (1998), 91-95.

[3] S. S. Dragomir, P. Cerone And A. Sofo, Some remarks on the midpoint rule in numerical integration, Studia Univ. Babes-Bolyai, Math., XLV (1) (2000), 63-74.

[4] S. S. Dragomir, P. Cerone And A. Sofo, Some remarks on the trapezoid rule in numerical integration, Indian J. Pure Appl. Math., 31 (5) (2000), 475-494.

[5] A. El FARISSi, Z. LATREUCH AND B. BALAIDI, Hadamard type inequalities for near convex functions, Gazeta Matematica Seria A, No. 1-2/2010.

[6] S. Furuichi AND H. R. Moradi, Advances in mathematical inequalities, De Gruyter, 2020.

[7] F. Kubo And T. Ando, Means of positive operators, Math. Ann., 264 (1980), 205-224.

[8] F. C. Mitroi-SYMeONidis, About the precision in Jensen-Steffensen inequality, An. Univ. Craiova Ser. Mat. Inform., 37 (4) (2010), 73-84.

[9] R. Pal, M. Singh, M. S. Moslehian And J. S. Aujla, A new class of operator monotone functions via operator means, Linear and Multilinear Algebra, 64 (12) (2016), 2463-2473. 